\title{
Growth differentiation factor 15 increases following oral glucose ingestion: effect of meal composition and obesity
}

\author{
Marie Helene Schernthaner-Reiter', Dominik Kasses', Christina Tugendsam', \\ Michaela RiedI' ${ }^{1}$, Slobodan Peric', Gerhard Prager ${ }^{2}$, Michael Krebs ${ }^{1}$, \\ Miriam Promintzer-Schifferl ${ }^{1}$, Martin Clodi ${ }^{1}$, Anton Luger ${ }^{1}$ and Greisa Vila ${ }^{1}$ \\ ${ }^{1}$ Clinical Division of Endocrinology and Metabolism, Department of Internal Medicine III, Medical \\ University of Vienna, Vienna, Austria and 'Division of General Surgery, Department of Surgery, \\ Medical University of Vienna, Vienna, Austria
}

Correspondence should be addressed to G Vila

Email

greisa.vila@meduniwien.ac.at

\begin{abstract}
Objective: Growth differentiation factor 15 (GDF15) is a cardiovascular biomarker belonging to the transforming growth factor- $\beta$ superfamily. Increased GDF15 concentrations are associated with insulin resistance, diabetes and obesity. We investigated the physiological effects of meal composition and obesity on the regulation of systemic GDF15 levels.

Design: Lean $(n=8)$ and obese $(n=8)$ individuals received a carbohydrate- or fat-rich meal, a $75 \mathrm{~g}$ oral glucose load (OGTT) or short-term fasting. OGTTs were performed in severely obese patients $(n=6)$ pre- and post-bariatric surgery. Methods: Circulating serum GDF15 concentrations were studied in lean and obese individuals in response to different meals, OGTT or short-term fasting, and in severely obese patients pre- and post-bariatric surgery. Regulation of GDF15 mRNA levels and protein release were evaluated in the human hepatic cell line HepG2.

Results: GDF15 concentrations steadily decrease during short-term fasting in lean and obese individuals.

Carbohydrate- and fat-rich meals do not influence GDF15, whereas an OGTT leads to a late increase in GDF15 levels.

The positive effect of OGTT on GDF15 levels is also preserved in severely obese patients, pre- and post-bariatric surgery. We further studied the regulation of GDF15 mRNA levels and protein release in HepG2, finding that glucose and insulin independently stimulate both GDF15 transcription and secretion.

Conclusion: In summary, high glucose and insulin peaks upregulate GDF15 transcription and release. The nutrientinduced increase in GDF15 levels depends on rapid glucose and insulin excursions following fast-digesting
\end{abstract} carbohydrates, but not on the amount of calories taken in.

\section{Introduction}

Growth differentiation factor 15 (GDF15) is a stressand inflammation-induced cytokine that belongs to the transforming growth factor- $\beta$ family (1). GDF15 is expressed in macrophages, liver, kidney, pancreas and in nearly all organs at low levels, and is upregulated following inflammation, tissue injury, remodelling and malignancy $(2,3)$.

In humans, increased circulating GDF15 concentrations are associated with ageing, C-reactive protein

www.eje-online.org DOI: 10.1530/EJE-16-0550
() 2016 European Society of Endocrinology Printed in Great Britain
(CRP) levels, tissue injury, insulin resistance, obesity and diabetes $(4,5,6,7)$. GDF15 levels are also independent predictors of future insulin resistance and diabetes (8). Furthermore, GDF15 was recently characterized as a central appetite regulator in mice, as $G d f 15^{-/-}$mice display obesity and increased food intake, which were rescued by administration of exogenous $\operatorname{GDF} 15(9,10)$. The associations of GDF15 with appetite, insulin resistance and inflammation have led to suggestions that GDF15 
may be a potential therapeutic target in obesity and diabetes (11).

In the clinical setting, GDF15 has gained attention as an emerging cardiovascular risk factor in all cohorts studied. Circulating GDF15 concentrations are associated with the incidence of cardiovascular events (12), mortality after cardiovascular events (13) and all-cause mortality (4). Being a marker of oxidative stress, endothelial dysfunction, atherosclerosis and inflammation, GDF15 provides independent prognostic information that extends beyond that of the classical cardiovascular risk factors $(1,14,15)$.

The use of GDF15 as a biomarker in clinical practice is limited by the scarce knowledge on mechanisms directly governing its regulation in humans. Stress, inflammation, glucose and insulin were found to stimulate GDF15 in translational studies $(1,16,17)$. Despite the well-established relationships between GDF15, insulin resistance and obesity, little is known about GDF15 regulation in response to nutrient intake in humans. We show here the profile of changes in serum GDF15 concentrations following an oral glucose load, two different meals (carbohydrate- vs fat-rich) and during short-term fasting, and its relationship with glucose and insulin levels in lean and obese subjects. In addition, we test the acute GDF15 response to an oral glucose load in severely obese patients pre- and postbariatric surgery. Furthermore, we study the independent and combined effects of glucose and insulin on GDF15 transcription and release in the human hepatic HepG2 cell line.

\section{Subjects and methods}

\section{Meal study: participants and study design}

This study included 8 lean (body mass index (BMI) $\leq 25 \mathrm{~kg}$ / $\left.\mathrm{m}^{2}\right)$ and 8 obese $\left(>30 \mathrm{~kg} / \mathrm{m}^{2}\right)$ subjects, and all participants gave written informed consent. BMI was calculated as weight $(\mathrm{kg}) /$ height $(\mathrm{m})^{2}$. The study protocol was approved by the institutional review board of the Medical University of Vienna. During the screening visit, a thorough medical history was taken and a detailed physical examination was performed. Blood samples were taken from an antecubital vein for the determination of haematological status, glucose, glycated haemoglobin (HbA1c) and clinical chemistry parameters.

Participants attended four study sessions, each separated by $>3$ days at the clinical research centre of the Division of Endocrinology at the Medical University of Vienna. The order of study sessions was randomized and single-blinded. Before admission, the participants were requested to refrain from consumption of alcohol or stimulating beverages containing xanthine derivatives for $12 \mathrm{~h}$, and to fast overnight. After admission between 7:50 and 9:30h, an i.v. cannula was placed in an antecubital vein and baseline blood samples were collected. Participants then received either (i) an oral glucose tolerance test (OGTT) containing a $75 \mathrm{~g}$ oral glucose load, $300 \mathrm{kcal}$, (Glucodrink, Roche); (ii) a carbohydraterich breakfast $(700 \mathrm{kcal}$, total carbohydrate content $121 \mathrm{~g}$, total fat content $13 \mathrm{~g}$, total protein content $22 \mathrm{~g}$ ); (iii) a fat-rich breakfast $(700 \mathrm{kcal}$, total carbohydrate content $53 \mathrm{~g}$, total fat content $45 \mathrm{~g}$, total protein content $22 \mathrm{~g}$ ), or (iv) nothing (fasting day). Blood samples were drawn at time-points 30, 60, 90, 120, 150 and $180 \mathrm{~min}$. During carbohydrate- and fat-rich breakfasts, at each time-point, participants rated their feelings of hunger and satiety on a $10 \mathrm{~cm}$ visual analogue scale.

\section{Bariatric surgery study: participants and study design}

Severely obese patients (BMI $>40 \mathrm{~kg} / \mathrm{m}^{2}$ ) who were planning to undergo Roux-en-Y gastric bypass surgery were referred to our department for preoperative endocrine evaluation. Six non-diabetic severely obese patients were included in this study. Before operation (pre-surgery) and 6-12 months postoperatively (post-surgery), a $75 \mathrm{~g}$ OGTT was performed and anthropometric measurements as well as haematology and clinical chemistry tests were performed. Blood samples were drawn at time-points 0, 60, 120 and $180 \mathrm{~min}$. All participants gave written informed consent. The study protocol was approved by the institutional review board of the Medical University of Vienna.

\section{Assays}

Glucose, HbA1c, lipid values, CRP and liver function tests were determined by standard certified protocols at the Institute of Medical and Chemical Laboratory Diagnostics. Samples for GDF15 and insulin determination were centrifuged at $4^{\circ} \mathrm{C}$ and $1500 \mathrm{~g}$ for $10 \mathrm{~min}$, and serum was immediately frozen at $-20^{\circ} \mathrm{C}$ until analysis. GDF15 serum levels were determined by quantitative sandwich ELISA (\#DGD150, R\&D Systems) with intra- and inter-assay CV of $<2.8$ and $<6 \%$, respectively. Insulin was determined using a commercially available RIA with inter- and intraassay CV of 2.5 and 3\%, respectively (Linco Research). 
The homeostasis model assessment insulin resistance index (HOMA-IR) was calculated as fasting glucose $(\mathrm{mg} / \mathrm{dL}) \times$ fasting insulin $(\mathrm{mU} / \mathrm{L}) / 405$.

\section{GDF15 mRNA expression and release in HepG2}

HepG2 cells were obtained from the American Type Culture Collection (Manassas, VA, USA) and kept under standard cell culture conditions $\left(37^{\circ} \mathrm{C}, 5 \% \mathrm{CO}_{2}\right)$. They were maintained in Dulbecco's modified Eagle's medium (DMEM), supplemented with 10\% foetal bovine serum (FBS), 2 mM L-glutamine (all from Gibco) and $50000 \mathrm{IU} / \mathrm{L}$ of penicillin and streptomycin (Lonza, Basel, Switzerland).

For stimulations, 150000 cells per well were seeded into 12-well plates and left overnight to recover. Cells were then placed in low-glucose DMEM (5.56 mM D-glucose) for $24 \mathrm{~h}$. For glucose stimulations cells were either placed in high-glucose DMEM (25 mM D-glucose) or low-glucose DMEM as a control. For insulin stimulations, cells were stimulated with $100 \mathrm{nM}$ human insulin (Sigma) in either low-glucose or high-glucose medium (glucose+insulin). Stimulations with glucose, insulin or glucose+insulin were performed for $4,8,24$ and $48 \mathrm{~h}$, as stated.

Supernatants were then completely removed and frozen at $-20^{\circ} \mathrm{C}$ for later analysis and cells were lysed for mRNA isolation, as described previously (18). Each time-point and experimental condition was performed in triplicate wells; triplicate control wells were performed separately for each time-point. GDF15 mRNA levels and supernatant GDF15 concentrations shown at different time-points correspond to the same experiment. Briefly, total RNA was isolated using Trizol (Invitrogen). RNA concentration and integrity were determined by UV spectrophotometry (Nanodrop 2000, Thermo Scientific). $1000 \mathrm{ng}$ were treated with DNAse and reverse transcribed using random primers and Superscript II (all Invitrogen) in a total of $20 \mu \mathrm{L}$ according to manufacturer's instructions. RT-qPCR was performed using $1 \mu \mathrm{L}$ cDNA transcript with $0.5 \mu \mathrm{L}$ 6-carboxyfluorescein (FAM)-labelled human GDF15 TaqMan probe (Applied Biosystems) and $0.5 \mu \mathrm{L}$ of VIClabelled glyceraldehyde-3-phosphate dehydrogenase $(G A P D H)$ probe in a duplex RT-qPCR reaction on an ABI Prism 7000 Cycler (Applied Biosystems). Relative expression was quantified using the $\Delta \Delta \mathrm{Ct}$ method. GDF15 concentration in cell culture supernatants was determined using a quantitative sandwich ELISA kit (R\&D Systems) as described (6). No significant differences were detected in relative GAPDH expression among all treatments at all different time-points, thereby excluding a significant confounding effect of glucose or insulin on cell proliferation.

\section{Statistics}

Data were tested for normality using Shapiro-Wilk tests. Normally distributed data are shown as mean \pm standard error of the mean (s.E.M.); non-normally distributed data are shown as median \pm interquartile range (IQR). Differences between means were tested by two-sided independent samples Student's $t$-tests or independent samples MannWhitney $U$ tests for nonparametric data. Paired samples were analysed by paired-samples $t$-tests or related-samples Wilcoxon signed-rank tests. The associations among variables were analysed using Spearman's rank correlation coefficients. The differences between the different study days, different time-points and between lean vs obese patients were assessed by repeated-measures analysis of variances (RM-ANOVA), with the term of interest being time, patient type or time*treatment as stated. Differences between means in cell culture experiments were analysed using two-way ANOVA followed by post-hoc testing by Dunnett's multiple comparisons test. $P$ values $<0.05$ were considered statistically significant. Data were analysed using SPSS Statistics (IBM).

\section{Results}

\section{Effect of glucose load and different meals on GDF15 concentrations}

Baseline clinical, metabolic and biochemical characteristics of the meal study participants are given in Table 1. Both lean and obese groups were comparable in age, height and gender distribution. While fasting glucose and HbA1c were within normal limits in the obese cohort, notably there were significant differences in fasting insulin, HOMA and CRP between lean and obese individuals.

The levels of serum GDF15, insulin and glucose during short-term fasting (Placebo), OGTT, carbohydraterich (Carb) and fat-rich (Fat) meals are depicted in Figs 1 and 2. During short-term fasting GDF15 concentrations showed a steady decrease in lean and obese subjects $(P<0.001$ and $P=0.012$, respectively, effect of time in RM-ANOVA, Fig. 1 and Supplementary Table 1, see section on supplementary data given at the end of this article). There was a trend towards a larger decrease in GDF15 levels during short-term fasting in lean compared with obese subjects, but this difference did not reach 
Table 1 Baseline clinical, metabolic and biochemical characteristics of participants of the meal study. Shown are mean (s.E.M.) for parametric data or median $( \pm \mathrm{IQR})$ for nonparametric data. Differences between means were tested with independent samples, Student's t-tests or independent samples Mann-Whitney $U$ tests for nonparametric data.

\begin{tabular}{lcc}
\hline & & Lean $(n=8)$ \\
\cline { 1 - 2 } Sex (male/female) & $5 / 3$ \\
Age (years) & $26.4(1.1)$ \\
Weight $(\mathrm{kg})$ & $71.4(6.4)$ \\
Height $(\mathrm{m})$ & $1.80(0.05)$ \\
BMI (kg/m²) & $21.7(0.9)$ \\
Waist circumference $(\mathrm{cm})$ & $77.1(4.9)$ \\
Hip circumference $(\mathrm{cm})$ & $91.3(3.5)$ \\
Waist/hip ratio & $0.90(0.06)$ \\
Fasting glucose (mg/dL) & $84.0(2.5)$ \\
Fasting insulin (mU/L) & $14.6(1.7)$ \\
HOMA-IR & $3.0(0.3)$ \\
HbA1c (\%) & $5.0(4.9-5.3)$ \\
Triglycerides (mg/dL) & $98.9(18.7)$ \\
Total cholesterol (mg/dL) & $182.0(5.1)$ \\
LDL (mg/dL) & $103.1(5.1)$ \\
HDL (mg/dL) & $59.1(4.6)$ \\
CRP (mg/dL) & $0.04(0.02-0.11)$ \\
Serum creatinine (mg/dL) & $0.97(0.04)$ \\
\hline
\end{tabular}

\begin{tabular}{ccl}
\hline Obese $(n=8)$ & & P value \\
\cline { 1 - 1 } $5 / 3$ & & \\
$26.7(1.3)$ & & 0.871 \\
$119.1(9.6)$ & & $0.001 * *$ \\
$1.78(0.06)$ & & 0.749 \\
$34.7(32.9-44.2)$ & & $<0.001 * * *$ \\
$120.3(5.9)$ & $<0.001 * * *$ \\
$123.0(4.9)$ & $<0.001 * * *$ \\
$0.98(0.03)$ & 0.287 \\
$85.9(2.6)$ & 0.607 \\
$29.5(3.3)$ & $0.001 * *$ \\
$6.4(0.9)$ & $0.003 * *$ \\
$5.4(0.1)$ & 0.161 \\
$99.5(71.3-128.3)$ & 0.574 \\
$178.8(9.1)$ & 0.760 \\
$110.4(9.3)$ & 0.504 \\
$45.9(3.5)$ & $0.040 *$ \\
$0.34(0.07)$ & $0.001 * *$ \\
$0.92(0.04)$ & 0.455 \\
\hline
\end{tabular}

$* P<0.05, * * P<0.005, * * * P<0.001$.

CRP, C-reactive protein; HbA1c, glycated haemoglobin; HDL, high-density lipoprotein; HOMA-IR, homeostatic model assessment insulin resistance; LDL, low-density lipoprotein.

significance $(P=0.074$, effect of time*treatment (lean vs obese) in RM-ANOVA). Conversely, during OGTT this steady decrease was inhibited and GDF15 increased back to baseline levels after the glucose load in both lean and obese individuals $(P=0.001$ and $P=0.021$, respectively, effect of time*treatment (placebo vs OGTT) in RM-ANOVA). The rise of GDF15 to baseline values was delayed compared with corresponding peaks of glucose and insulin and only became evident after $120 \mathrm{~min}$ $(P<0.001$ between time-point $120 \mathrm{~min}$ and time-point $180 \mathrm{~min}$ in Bonferroni-corrected pairwise comparisons). GDF15 levels during fat- and carbohydrate-rich meals were not significantly different from placebo in both lean and obese groups.
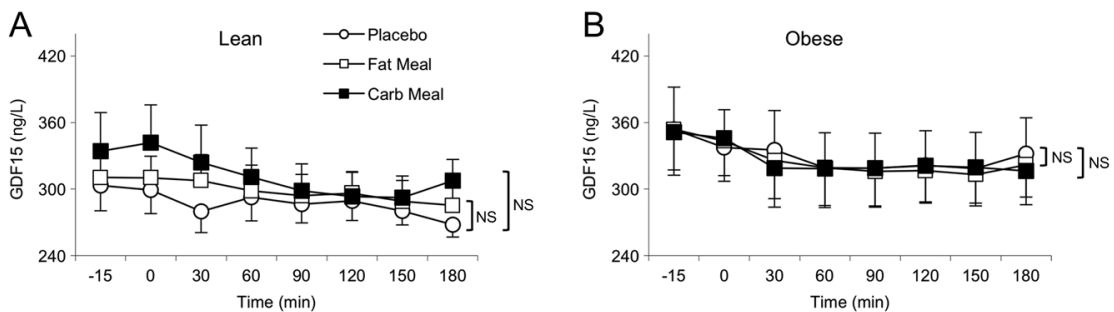

Figure 1

Profile of changes in GDF15 in lean and obese subjects. (A and B) Serum GDF15 concentrations over $180 \mathrm{~min}$ in response to short-term fasting (white circles) or isocaloric high fat (Fat meal, white squares) or high carbohydrate (Carb meal, black squares) breakfasts in lean $(A, n=8)$ and obese probands $(B, n=8)$. ( $C$ and $D)$ Serum GDF15 concentrations over $180 \mathrm{~min}$ in response to short-term fasting (white circles) or a $75 \mathrm{~g}$ oral glucose tolerance test (OGTT, black circles) in lean (C, $n=8)$
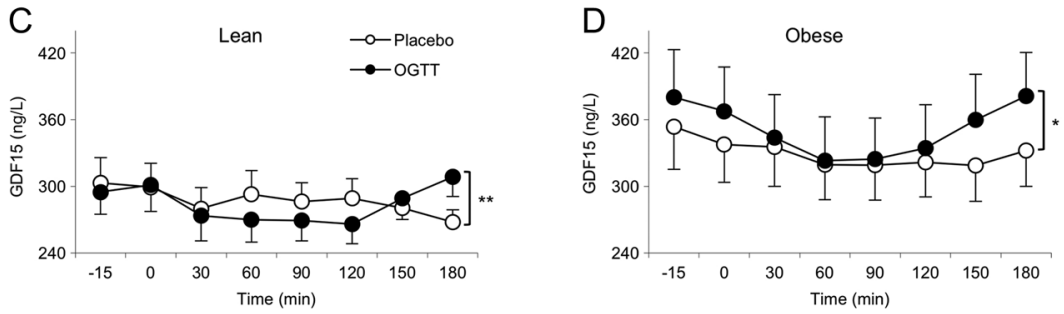
and obese probands ( $D, n=8)$. Shown are the mean serum concentrations, error bars represent the S.E.M. Differences between treatments were analysed using repeated-measures ANOVA. * $P<0.05$, $* * P<0.005$, NS, not significant. 
A
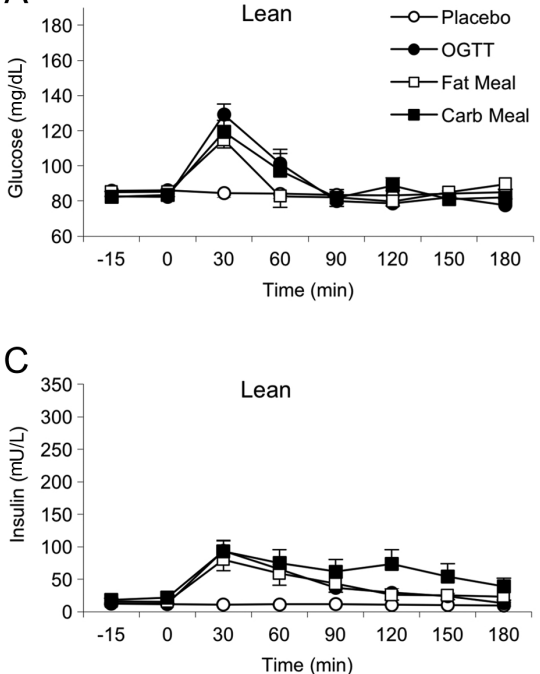

B
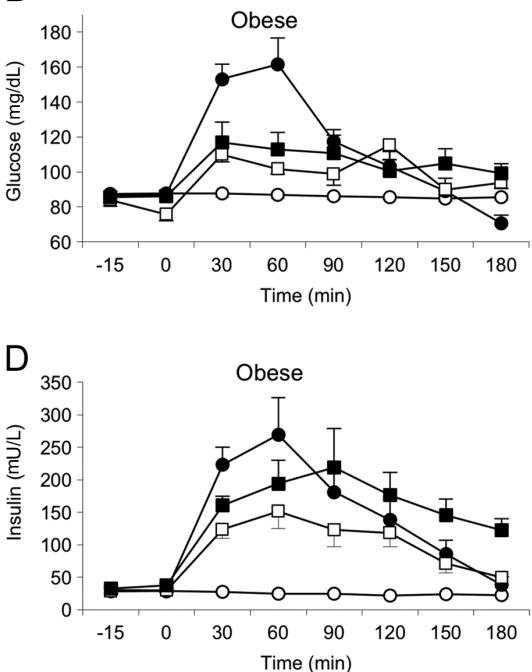

Figure 2

Serum glucose ( $A$ and $B$ ) and insulin ( $C$ and $D$ ) concentrations over $180 \mathrm{~min}$ in response to short-term fasting (white circles), a $75 \mathrm{~g}$ oral glucose load (OGTT, black circles) or isocaloric high fat (Fat meal, white squares) or high carbohydrate (Carb meal, black squares) breakfasts in lean ( $A$ and $B, n=8)$ and obese probands ( $C$ and $D, n=8$ ). Shown are the mean serum concentrations, error bars represent the S.E.M.
OGTT, carbohydrate-rich meals and fat-rich meals led to significantly increased glucose and insulin levels when compared with placebo in all groups (Supplementary Tables 2 and 3). The profile of changes in circulating glucose following a carbohydrate-rich meal was significantly different than following an OGTT, with higher glucose peaks reached during the OGTT ( $P=0.009$, effect of time*treatment (OGTT vs carb meal) in RM-ANOVA for all patients). Glucose and insulin concentrations achieved during the OGTT were significantly higher in obese vs lean patients $(P=0.005$ for glucose differences in lean vs obese patients and $P=0.002$ for insulin differences in lean vs obese patients).

We further tested the relationship of GDF15 levels with subjective ratings of appetite and found that the AUC of GDF15 during short-term fasting positively correlates with hunger in lean subjects (Supplementary Table 4). No other correlations were found between hunger/satiety scores and GDF15, insulin or glucose.

Table 2 Clinical characteristics of obese patients before (pre-OP) and after bariatric surgery (post-OP). Shown are mean (s.E.M.) for parametric data or median $( \pm \mathrm{IQR})$ for nonparametric data. Differences between means were tested with paired samples, Student's $t$-tests or related samples Wilcoxon signed-rank tests for nonparametric data.

\begin{tabular}{|c|c|c|c|}
\hline & Pre-OP $(n=6)$ & Post-OP $(n=6)$ & $P$ value \\
\hline Sex (male/female) & $1 / 5$ & & \\
\hline Age (years) & $43.2(2.1)$ & $43.8(2.1)$ & \\
\hline Weight (kg) & $138.1(14.8)$ & $102.9(12.3)$ & $<0.001 * * *$ \\
\hline Height (m) & $165.8(4.0)$ & & \\
\hline BMI $\left(\mathrm{kg} / \mathrm{m}^{2}\right)$ & $49.3(3.2)$ & 36.7 (2.9) & $<0.001 * * *$ \\
\hline Waist circumference $(\mathrm{cm})^{\dagger}$ & $137.0(7.2)$ & $111.0(8.9)$ & $0.005^{*}$ \\
\hline Hip circumference $(\mathrm{cm})^{\dagger}$ & $151.5(9.6)$ & $121.0(9.4)$ & $0.002 * *$ \\
\hline Waist/hip ratio* & $0.90(0.04)$ & $0.92(0.03)$ & 0.709 \\
\hline Fasting glucose $(\mathrm{mg} / \mathrm{dL})$ & $98.3(5.2)$ & $84.2(2.7)$ & $0.016^{*}$ \\
\hline Fasting insulin (mU/L) & $28.5(5.0)$ & $10.1(1.3)$ & $0.008^{*}$ \\
\hline HOMA-IR & $6.9(1.2)$ & $2.1(0.3)$ & $0.007 *$ \\
\hline HbA1c (\%) & $5.8(0.10)$ & $5.2(0.08)$ & $0.002 * *$ \\
\hline Triglycerides (mg/dL) & $112.5(96.0-145.0)$ & $93.7(3.6)$ & $0.046^{*}$ \\
\hline Total cholesterol (mg/dL) & $191.2(10.4)$ & $147.5(7.4)$ & $0.001 * *$ \\
\hline $\mathrm{LDL}(\mathrm{mg} / \mathrm{dL})$ & $118.2(7.9)$ & $85.4(9.0)$ & $0.008 *$ \\
\hline $\mathrm{HDL}(\mathrm{mg} / \mathrm{dL})$ & $48.2(2.4)$ & $43.3(3.0)$ & 0.250 \\
\hline C-reactive protein $(\mathrm{mg} / \mathrm{dL})^{\dagger}$ & $1.51(0.28)$ & $0.86(0.31)$ & $0.011 *$ \\
\hline Serum creatinine $(\mathrm{mg} / \mathrm{dL})$ & $0.77(0.04)$ & $0.72(0.02)$ & 0.201 \\
\hline
\end{tabular}

${ }^{\dagger}$ one measurement missing. $* P<0.05, * * P<0.005, * * * P<0.001$.

CRP, C-reactive protein; HbA1C, glycated haemoglobin; HDL, high-density lipoprotein; HOMA-IR, homeostatic model assessment insulin resistance; LDL, low-density lipoprotein. 
A

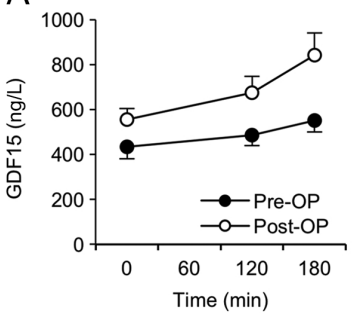

B

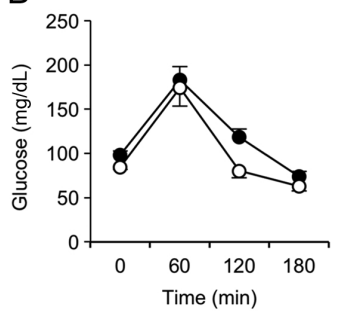

C

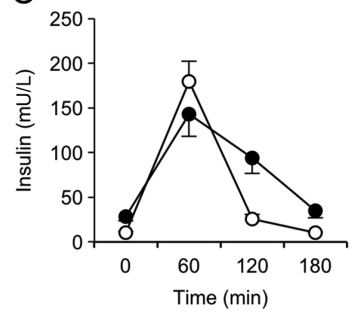

Figure 3

Effects of a $75 \mathrm{~g}$ oral glucose load on serum GDF15 (A), serum glucose (B) and serum insulin (C) concentrations in eight patients before bariatric surgery (black circles) and post-surgery (white circles). Data shown are the mean, error bars represent the S.E.M.

\section{Glucose-induced changes in GDF15 concentrations before and after bariatric surgery}

Effects of an oral glucose load on six severely obese patients were studied before and after bariatric surgery (Roux-en-Y gastric bypass). Pre- and post-surgery clinical, metabolic and biochemical characteristics of patients are given in Table 2 . The average weight loss after surgery was $35.2 \mathrm{~kg}$. Significant decreases were seen in weight, BMI, waist circumference, fasting glucose, total cholesterol, LDL cholesterol and in CRP after surgery.

The profiles of changes in serum GDF15, glucose and insulin after an oral glucose load performed before and after gastric bypass surgery are shown in Fig. 3. The significant increase in serum GDF15 over time following an oral glucose load was also sustained after bariatric surgery ( $P=0.002$ for OGTTs before surgery and $P=0.044$ for OGTTs after surgery, effect of time in RM-ANOVA). Surgery did not significantly impact the profile of changes in GDF15 following OGTT $(P=0.138$, effect of time*treatment in RM-ANOVA). The OGTT-induced changes in glucose concentrations were also similar pre- and post-surgery $(P=0.083$, effect of time*treatment in RM-ANOVA). There was a significant difference in the profile of changes in insulin concentrations during OGTTs pre- and post-surgery $(P<0.001$, effect of time*treatment in RM-ANOVA) with lower baseline concentrations but higher glucose-induced peaks reached after gastric bypass surgery.

\section{GDF15 transcription and release are stimulated independently by glucose and insulin}

At the mRNA level, both glucose and insulin significantly stimulate GDF15 after $4 \mathrm{~h}$ (Fig. 4A). The combination of glucose and insulin significantly increases GDF15 mRNA levels after 4 and $8 \mathrm{~h}$; conversely, after $24 \mathrm{~h}$ a strong counterregulation leads to the decrease of GDF15 transcription by both agents (Fig. 4A). GDF15 protein release is also increased by glucose and insulin by approximately $50 \%$ at 4, 8, 24 and $48 \mathrm{~h}$ (Fig. 4B); interestingly, this effect is predominantly driven by insulin in the earlier phase, with
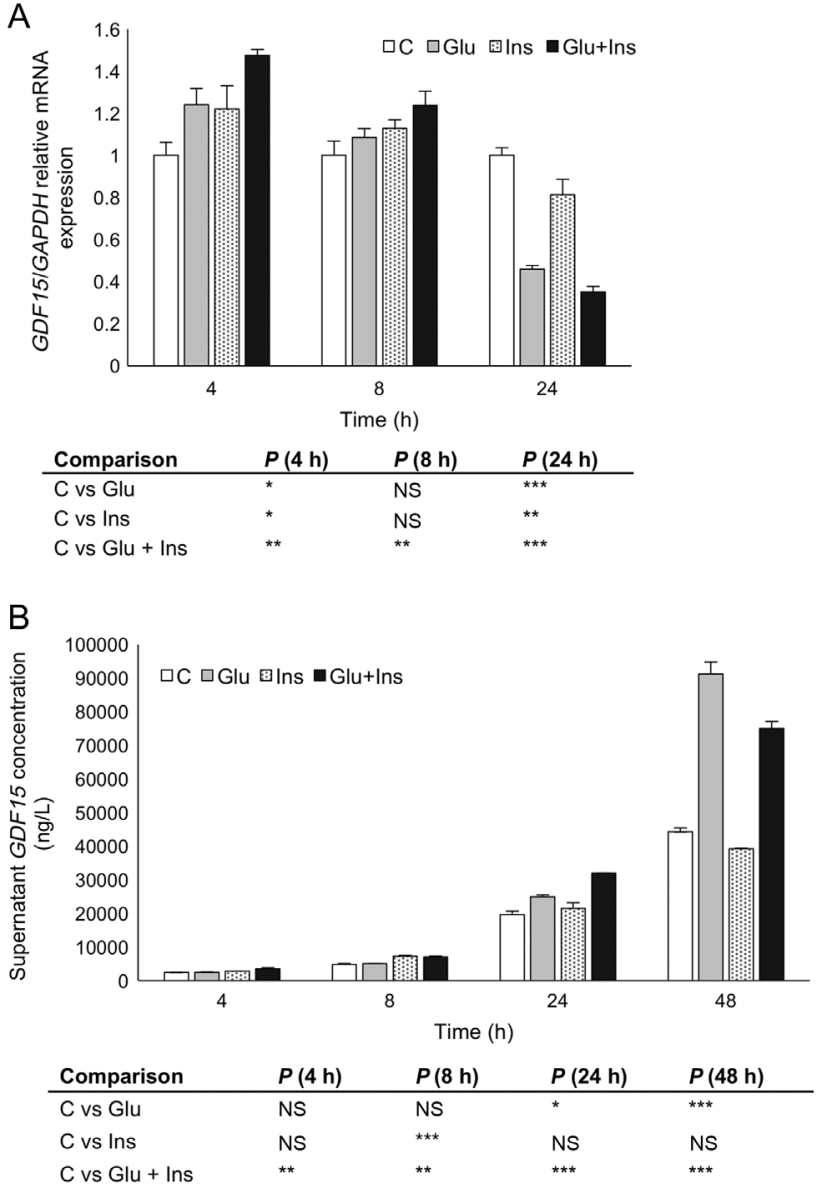

Figure 4

GDF15 transcription and release in response to glucose and insulin in HepG2. HepG2 cells were stimulated with glucose (Glu), insulin (Ins) or glucose and insulin (Glu +Ins) and compared with control (C) for 4, 8, 24 or 48h. GDF15 mRNA expression was analysed by RT-qPCR (A) and supernatant GDF15 levels were measured by ELISA (B) in the same experiment and the same well. Each time-point and experimental setting, including the controls, were performed in triplicate wells. Differences between means were tested with two-way ANOVA and significances for each comparison are given in the corresponding tables below, error bars indicate S.E.M. ${ }^{*} P>0.05$, $* * P>0.005, * * * P>0.001, \mathrm{NS}$, not significant. 
a peak at around $8 \mathrm{~h}$, and by glucose in the later phase (up to $48 \mathrm{~h}$ ). No interactions between glucose and insulin on GDF15 transcription and release were detected by two-way ANOVA (data not shown).

\section{Discussion}

We show here that GDF15 levels steadily decrease during short-term fasting in lean and healthy obese individuals, while an oral glucose load inhibits this initial decrease causing a late rise in GDF15 back to baseline levels. The stimulatory effect of glucose load on GDF15 concentrations is also preserved in severely obese patients before and after bariatric surgery. In vitro, glucose and insulin strongly biphasically stimulate both GDF15 transcription and protein secretion in the human hepatic cell line HepG2.

GDF15 was recently demonstrated to be regulated in a diurnal fashion, with a peak around midnight and nadir around noon in most subjects; no significant effect of different meals on GDF15 was observed (19). Our data confirm the decrease of GDF15 during short-term fasting, and no significant difference between short-term fasting and a carbohydrate- or fat-rich breakfast. We performed all testing during the morning hours; taken together, these results corroborate that the decline in GDF15 concentrations in the morning hours is likely to be, at least in part, influenced by meal-independent diurnal variations.

GDF15 is increased in obesity and type 2 diabetes mellitus $(6,8,20)$ and predicts future insulin resistance and diabetes (8). Here, we show that GDF15 significantly increases following an oral glucose load in lean and healthy obese subjects, as well as in severely obese patients before and after bariatric surgery. OGTT, despite containing fewer calories, led to significantly higher circulating glucose peak concentrations when compared with both meals in our cohort, suggesting that the difference in GDF15 response to an oral glucose load vs a carbohydrate-rich meal may be due to the amplitude of glucose excursions. Thus, fast-digesting carbohydrates would be expected to lead to higher GDF15 levels than other nutrients.

The GDF15 response to OGTT did not significantly differ between lean and obese subjects, while obese participants had significantly higher glucose and insulin excursions, thus suggesting that the glucose-dependent GDF15 response may be relatively blunted in obese individuals. In vitro, GDF15 expression is stimulated by high glucose in human endothelial cells, preventing apoptosis (16). In vivo, circulating GDF15 concentrations increase during euglycaemic hyperinsulinaemic clamps (17). We show here that GDF15 transcription and release in HepG 2 cells are significantly stimulated by glucose and insulin. The different time-dependent peaks of secreted GDF15 levels by glucose and insulin suggest that both glucose and insulin stimulate GDF15 transcription and release by different mechanisms; however, they may share a common pathway or endpoint as no interaction effects were detected. Furthermore, the regulation of GDF15 transcription and release are divergent, where transcription is downregulated after $24 \mathrm{~h}$ while release is still highly stimulated, suggesting that the regulation of systemic GDF15 levels are substantially influenced by posttranscriptional factors that may include mRNA stability or intracellular storage of mature GDF15.

GDF15 is expressed in placenta, macrophages, liver, pancreas, kidney and endothelial cells (2). In mice and in liver cell lines, hepatic GDF15 transcription is stimulated within $1-3 \mathrm{~h}$ of induction of liver injury (21). As the liver is also a key regulatory organ in insulin sensitivity and glucose disposal, we explored GDF15 regulation in response to changes in glucose and insulin in the HepG2 liver cell line. The glucose-dependent GDF15 release reported here takes place after around $120 \mathrm{~min}$, and is therefore in contrast to the rapid first-phase insulin peak or the secretion patterns of classical appetite regulators such as ghrelin and PYY, with maximum changes reached at around 30-60 min after glucose load $(22,23)$; an immediate induction of GDF15 release in humans has not been reported. We can therefore not exclude that the glucose-triggered GDF15 release is mediated via another glucose-dependent messenger; more research is necessary to characterize the cellular mechanisms of GDF15 release, and to identify the main source of human serum GDF15. The short-term stimulatory effects of glucose and insulin on GDF15 release might also contribute to the pathophysiology of the long-term increase in GDF15 concentrations in patients with insulin resistance and type 2 diabetes $(6,20)$.

During short-term fasting, the AUC of GDF15 correlates with hunger in lean, but not in obese subjects. To our knowledge, this is the first study to relate GDF15 levels to the subjective feelings of appetite. GDF15 was recently suggested to be a central appetite regulator in mice $(9,10)$. Furthermore, in GDF15 transgenic mice, glucose tolerance is improved, potentially due to improved insulin sensitivity (24). In humans, the mechanisms by which GDF15 influences appetite and body weight seem to be more complex: GDF15 levels are increased not only in obese patients, but also in 
patients with anorexia nervosa, in patients with cancer cachexia and following diet- or bariatric surgery-induced weight loss $(6,11,17)$. In contrast to recent murine data, there does not seem to be a strong relationship between GDF15 levels and appetite during meals (19); however, the increase in GDF15 after all types of weight loss suggests a relationship with energy homeostasis in the long term. On the other side, the positive relationship between GDF15 and insulin resistance, diabetes and obesity might be the pathophysiologic consequence of the long-term increases in glucose and insulin levels. Thus, the paradox of increased GDF15 levels not only in obesity, but also after weight loss, might be explained by the fact that nutrient availability (high glucose concentrations) and weight loss (inducing compensatory changes in appetite-regulatory mechanisms) modify GDF15 secretion either using different mechanisms (hormonal, neural) or by acting in different organs.

In summary, here we show that glucose and insulin are novel regulators of GDF15 transcription and release into the circulation. In vivo, a glucose load leads to increased GDF15 concentrations not only in lean, but also in obese subjects. These short-term effects of glucose and insulin might also contribute to the long-term increases in GDF15 levels observed in patients with insulin resistance and diabetes.

\section{Supplementary data}

This is linked to the online version of the paper at http://dx.doi.org/10.1530/ EJE-16-0550.

\section{Declaration of interest}

The authors declare that there is no conflict of interest that could be perceived as prejudicing the impartiality of the research reported.

\section{Funding}

This study was supported by the Medical-Scientific Fund of the Mayor of Vienna to M R.

\section{Author contribution statement}

G V, C T and A L designed the study. M H S R, D K, M R, S P, G P, M K, $M P S$ and M C performed research. G V, A L and M H S R analysed the data and contributed to discussion. M H S R wrote the initial version of the manuscript. All authors critically revised the manuscript and approved its final version.

\section{Acknowledgements}

We thank Astrid Hofer and Liliana-Imi Ionasz for excellent technical assistance.

\section{References}

1 Bootcov MR, Bauskin AR, Valenzuela SM, Moore AG, Bansal M, He XY, Zhang HP, Donnellan M, Mahler S, Pryor K et al. MIC-1, a novel macrophage inhibitory cytokine, is a divergent member of the TGF-beta superfamily. PNAS 199794 11514-11519. (doi:10.1073/ pnas.94.21.11514)

2 Fairlie WD, Moore AG, Bauskin AR, Russell PK, Zhang HP \& Breit SN MIC-1 is a novel TGF-beta superfamily cytokine associated with macrophage activation. Journal of Leukocyte Biology 199965 2-5.

3 Su AI, Wiltshire T, Batalov S, Lapp H, Ching KA, Block D, Zhang J, Soden R, Hayakawa M, Kreiman G et al. A gene atlas of the mouse and human protein-encoding transcriptomes. PNAS 2004101 6062-6067. (doi:10.1073/pnas.0400782101)

4 Wiklund FE, Bennet AM, Magnusson PK, Eriksson UK, Lindmark F, Wu L, Yaghoutyfam N, Marquis CP, Stattin P, Pedersen NL et al. Macrophage inhibitory cytokine-1 (MIC-1/GDF15): a new marker of all-cause mortality. Aging Cell 20109 1057-1064. (doi:10.1111/j.14749726.2010.00629.x)

5 Lind L, Wallentin L, Kempf T, Tapken H, Quint A, Lindahl B, Olofsson S, Venge P, Larsson A, Hulthe J et al. Growth-differentiation factor-15 is an independent marker of cardiovascular dysfunction and disease in the elderly: results from the Prospective Investigation of the Vasculature in Uppsala Seniors (PIVUS) Study. European Heart Journal 200930 2346-2353. (doi:10.1093/eurheartj/ehp261)

6 Vila G, Riedl M, Anderwald C, Resl M, Handisurya A, Clodi M, Prager G, Ludvik B, Krebs M \& Luger A. The relationship between insulin resistance and the cardiovascular biomarker growth differentiation factor-15 in obese patients. Clinical Chemistry 201157 309-316. (doi:10.1373/clinchem.2010.153726)

7 Kahli A, Guenancia C, Zeller M, Grosjean S, Stamboul K, Rochette L, Girard C \& Vergely C. Growth differentiation factor-15 (GDF-15) levels are associated with cardiac and renal injury in patients undergoing coronary artery bypass grafting with cardiopulmonary bypass. PLOS ONE 20149 e105759. (doi:10.1371/journal. pone.0105759)

8 Kempf T, Guba-Quint A, Torgerson J, Magnone MC, Haefliger C, Bobadilla M \& Wollert KC. Growth differentiation factor 15 predicts future insulin resistance and impaired glucose control in obese nondiabetic individuals: results from the XENDOS trial. European Journal of Endocrinology 2012167 671-678. (doi:10.1530/EJE-12-0466)

9 Tsai VW, Macia L, Johnen H, Kuffner T, Manadhar R, Jorgensen SB, Lee-Ng KK, Zhang HP, Wu L, Marquis CP et al. TGF-b superfamily cytokine MIC-1/GDF15 is a physiological appetite and body weight regulator. PLOS ONE 20138 e55174. (doi:10.1371/journal. pone.0055174)

10 Johnen H, Lin S, Kuffner T, Brown DA, Tsai VW, Bauskin AR, Wu L, Pankhurst G, Jiang L, Junankar S et al. Tumor-induced anorexia and weight loss are mediated by the TGF-beta superfamily cytokine MIC-1. Nature Medicine 200713 1333-1340. (doi:10.1038/nm1677)

11 Tsai VW, Lin S, Brown DA, Salis A \& Breit SN. Anorexia-cachexia and obesity treatment may be two sides of the same coin: role of the TGF-b superfamily cytokine MIC-1/GDF15. International Journal of Obesity 201540 193-197. (doi:10.1038/ijo.2015.242)

12 Brown DA, Breit SN, Buring J, Fairlie WD, Bauskin AR, Liu T \& Ridker PM. Concentration in plasma of macrophage inhibitory cytokine-1 and risk of cardiovascular events in women: a nested case-control study. Lancet 2002359 2159-2163. (doi:10.1016/S01406736(02)09093-1)

13 Kempf T, Bjorklund E, Olofsson S, Lindahl B, Allhoff T, Peter T, Tongers J, Wollert KC \& Wallentin L. Growth-differentiation factor-15 improves risk stratification in ST-segment elevation myocardial infarction. European Heart Journal 200728 2858-2865. (doi:10.1093/ eurheartj/ehm465)

14 Han ES, Muller FL, Perez VI, Qi W, Liang H, Xi L, Fu C, Doyle E, Hickey $\mathrm{M}$, Cornell $\mathrm{J}$ et al. The in vivo gene expression signature 
of oxidative stress. Physiological Genomics 200834 112-126. (doi:10.1152/physiolgenomics.00239.2007)

15 Rohatgi A, Patel P, Das SR, Ayers CR, Khera A, Martinez-Rumayor A, Berry JD, McGuire DK \& de Lemos JA. Association of growth differentiation factor-15 with coronary atherosclerosis and mortality in a young, multiethnic population: observations from the Dallas Heart Study. Clinical Chemistry 201258 172-182. (doi:10.1373/ clinchem.2011.171926)

16 Li J, Yang L, Qin W, Zhang G, Yuan J \& Wang F. Adaptive induction of growth differentiation factor 15 attenuates endothelial cell apoptosis in response to high glucose stimulus. PLoS ONE 20138 e65549. (doi:10.1371/journal.pone.0065549)

17 Karczewska-Kupczewska M, Kowalska I, Nikolajuk A, Adamska A, Otziomek E, Gorska M \& Straczkowski M. Hyperinsulinemia acutely increases serum macrophage inhibitory cytokine- 1 concentration in anorexia nervosa and obesity. Clinical Endocrinology 201276 46-50. (doi:10.1111/j.13652265.2011.04139.x)

18 Schernthaner-Reiter MH, Kiefer F, Zeyda M, Stulnig TM, Luger A \& Vila G. Strong association of serum- and glucocorticoid-regulated kinase 1 with peripheral and adipose tissue inflammation in obesity. International Journal of Obesity 201539 1143-1150. (doi:10.1038/ ijo.2015.41)

19 Tsai VW, Macia L, Feinle-Bisset C, Manandhar R, Astrup A, Raben A, Lorenzen JK, Schmidt PT, Wiklund F, Pedersen NL et al. Serum levels of human MIC-1/GDF15 vary in a diurnal pattern, do not display a profile suggestive of a satiety factor and are related to BMI. PLOS ONE 201510 e0133362. (doi:10.1371/journal. pone.0133362)

20 Dostalova I, Roubicek T, Bartlova M, Mraz M, Lacinova Z, Haluzikova D, Kavalkova P, Matoulek M, Kasalicky M \& Haluzik M. Increased serum concentrations of macrophage inhibitory cytokine-1 in patients with obesity and type 2 diabetes mellitus: the influence of very low calorie diet. European Journal of Endocrinology 2009161 397-404. (doi:10.1530/EJE-09-0417)

21 Hsiao EC, Koniaris LG, Zimmers-Koniaris T, Sebald SM, Huynh TV $\&$ Lee SJ. Characterization of growth-differentiation factor 15 , a transforming growth factor beta superfamily member induced following liver injury. Molecular and Cellular Biology 200020 3742-3751. (doi:10.1128/MCB.20.10.3742-3751.2000)

22 Shiiya T, Nakazato M, Mizuta M, Date Y, Mondal MS, Tanaka M, Nozoe S, Hosoda H, Kangawa K \& Matsukura S. Plasma ghrelin levels in lean and obese humans and the effect of glucose on ghrelin secretion. Journal of Clinical Endocrinology and Metabolism 200287 240-244. (doi:10.1210/jcem.87.1.8129)

23 Kim BJ, Carlson OD, Jang HJ, Elahi D, Berry C \& Egan JM. Peptide $\mathrm{YY}$ is secreted after oral glucose administration in a gender-specific manner. Journal of Clinical Endocrinology and Metabolism 200590 6665-6671. (doi:10.1210/jc.2005-0409)

24 Macia L, Tsai VW, Nguyen AD, Johnen H, Kuffner T, Shi YC, Lin S, Herzog H, Brown DA, Breit SN et al. Macrophage inhibitory cytokine 1 (MIC-1/GDF15) decreases food intake, body weight and improves glucose tolerance in mice on normal \& obesogenic diets. PLOS ONE 20127 e34868. (doi:10.1371/journal.pone.0034868)

Received 26 June 2016

Revised version received 31 August 2016

Accepted 19 September 2016 